\title{
Dynamic infrared thermography and smartphone thermal imaging as an adjunct for preoperative, intraoperative, and postoperative perforator free flap monitoring
}

\author{
Geoffrey G. Hallock \\ Division of Plastic Surgery, St. Luke's Hospital, Sacred Heart Campus, Allentown, Pennsylvania 18103, USA.
}

Correspondence to: Dr. Geoffrey G. Hallock, Division of Plastic Surgery, St. Luke's Hospital, Sacred Heart Campus, 1230 South Cedar Crest Boulevard, Suite 306 Allentown, Pennsylvania 18103, USA. E-mail: gghallock@hotmail.com

\begin{abstract}
How to cite this article: Hallock GG. Dynamic infrared thermography and smartphone thermal imaging as an adjunct for preoperative, intraoperative, and postoperative perforator free flap monitoring. Plast Aesthet Res 2019;6:29.

http://dx.doi.org/10.20517/2347-9264.2019.029
\end{abstract}

Received: 14 Sep 2019 First Decision: 8 Nov 2019 Revised: 24 Nov 2019 Accepted: 2 Dec 2019 Published: 17 Dec 2019

Science Editor: Matthew L. lorio Copy Editor: Jing-Wen Zhang Production Editor: Tian Zhang

\begin{abstract}
Aim: The versatile application of perforator free flaps for coverage of any extremity has been well proven. Often, a "freestyle"-like approach is used to design these flaps, as conventional imaging techniques for perforator identification may be too expensive or unavailable. As will be demonstrated, the recent application of a thermal imaging camera using a smartphone is a cheaper and therefore more universal means to better identify the requisite perforators upon which a free flap can be designed and then monitored.
\end{abstract}

Methods: Smartphone thermography can be used on any patient preoperatively to identify preferable perforators or vascular network "hot spots" within the desired donor site territory. Intraoperative management of the choice of perforators and subsequent flap dissection can be similarly facilitated. Intermittent postoperative monitoring based on changes of the thermal image color palette will provide a comparison that can be used to determine if perfusion across the microanastomosis is sustained.

Results: An overview of how to use a smartphone in concert with a thermal imaging camera is outlined. Dynamic infrared thermography represents a thermal stress necessary with a smartphone to better identify donor site "hot spots".

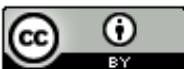

(C) The Author(s) 2019. Open Access This article is licensed under a Creative Commons Attribution 4.0 International License (https://creativecommons.org/licenses/by/4.0/), which permits unrestricted use, sharing, adaptation, distribution and reproduction in any medium or format, for any purpose, even commercially, as long as you give appropriate credit to the original author(s) and the source, provide a link to the Creative Commons license, and indicate if changes were made. 
Conclusion: Smartphone thermography is an inexpensive and expeditious means for identification of "hot spots" that correlate with perforators that would suffice to insure perfusion to a free perforator flap. However, since perforator caliber and course cannot be determined, this should be considered to be only a complementary adjunct for conventional methods. Nevertheless, its simplicity will overall improve the safer design, harvest, and subsequent monitoring of free flaps.

Keywords: Smartphone, thermography, thermal image camera, perforator free flap, microvascular tissue transfer, monitor

\section{INTRODUCTION}

Thermal imaging is in reality not an esoteric principle of physics that should be feared, as multiple roles are already commonplace as this is the basis for night vision utilized by the military, or in civilian life a means to detect heat loss sources from construction sites or something as prevalent in the hospital setting as preexisting deep-tissue pressure injuries ${ }^{[1]}$. It is amazing that more than 30 years ago, Theuvenet $e$ al $^{[2]}$ actually applied this concept for assessment of perforator arteries of fasciocutaneous and musculocutaneous flaps! How this is possible is the intriguing aspect, and requires some understanding of human biophysiology, particularly as regards our homeostatic mechanisms for maintaining body temperature equilibrium.

Many factors actively influence skin temperature; however, assuming all else is constant, the principal mechanism for heat dissipation is via radiative heat loss from the skin to the environment ${ }^{[3,4]}$. The medium used to transport heat throughout the body is blood circulation, thus a good correlation exists between the given skin temperature and the quality of its skin perfusion ${ }^{[3]}$. From a basic physics standpoint, what is perceived as heat loss by the body is really infrared radiation whose wavelength falls within the non-visible range $(700-1 \mathrm{~mm})$ within the electromagnetic spectrum ${ }^{[5]}$. The quantity of infrared radiation that is emitted will be manifested by increments in alterations of the skin temperature observed, and this is directly correlated to variations in the cutaneous blood flow ${ }^{[5,6]}$.

A thermal imaging camera will be essential for the desired analysis of the given cutaneous infrared emission, and more importantly variations in flap perfusion. Muntean et al..$^{[7]}$ correctly pointed out that professional cameras are superior in their ability to do this, as these can pick-up temperature differences of as little as $0.04^{\circ} \mathrm{C}$ that can be modulated by the cardiac rhythm itself ${ }^{[8]}$ ! Such diminutive variations will allow detection of skin "hot spots", where greater heat is being emitted and most likely via a dominant perforator, as well as the degree of thermal extension into the surrounding vascular network so served, which today we might call the perforasome of that perforator ${ }^{[3-6,8-10]}$. Unfortunately, the widespread acquisition of this technology has been hampered by the extreme cost of these cameras.

Fortunately, however, technology has moved on, as today everyone has a smartphone. Incredibly inexpensive miniature thermal imaging cameras [FLIR ONE Pro (FLIR Systems, Inc., Willsonville, Oregon), FLIR.com/FLIRONE/Start] are available for $\sim 1 / 100$ th the cost of a professional camera, or just a few hundred dollars. This may be plugged into any type of smartphone. Using an app provided by the vendor, rapid real time thermogram still images or videos can be digitally merged with the visible light camera photograph from the smartphone ${ }^{[11]}$. Although the smartphone provides a lower resolution image and narrower temperature detection range than the more expensive professional cameras ${ }^{[10,11]}$, Pereira ${ }^{[12]}$ insisted that, for applications such as for perforator flaps, the accuracy thus far has proven to be enough.

Because of the lesser sensitivity of the smartphone thermal imaging camera, an initial thermal stress or "cold challenge" not required by the professional cameras will be more informative. This is why dynamic infrared thermography (DIRT) is a preferred adjunct ${ }^{[3-5,13,14]}$. DIRT is simplest done preoperatively using Muntean's method of spraying the proposed flap donor site with isopropyl alcohol followed by accelerated 


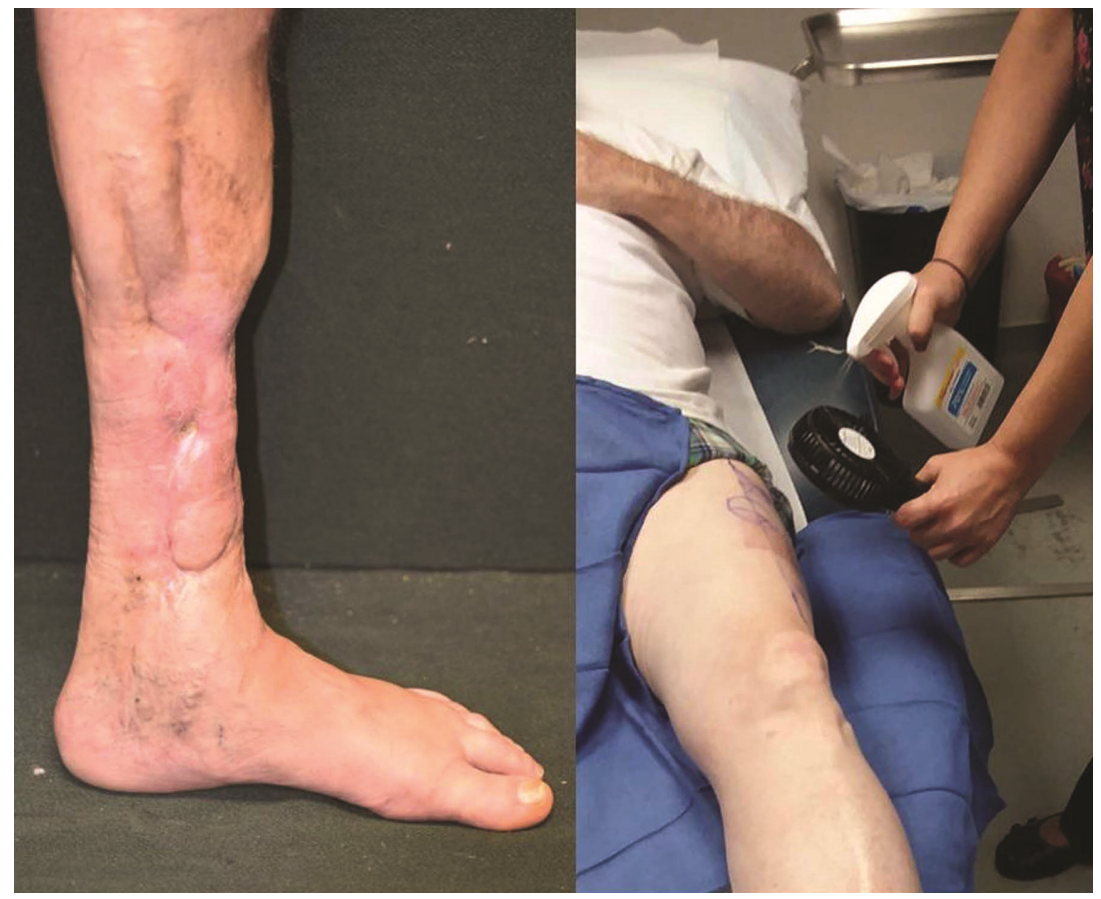

Figure 1. Preoperative case example. Chronic skin graft breakdown and drainage from medial left lower leg, 20 years following a motorcycle accident that at that time had a failed free flap and then a cross-leg flap (left), thermal stress on left anterolateral thigh donor site induced by isopropyl alcohol spray with fan to accelerate evaporation and cooling (right)

evaporation for cooling with a high speed portable fan ${ }^{[5]}$. Intraoperatively, a bag of ice instead can be used. This bedside test requires only a few minutes as the site rewarms using the thermal images observed as a valuable guide for further perforator identification with an audible Doppler probe or color Duplex ultrasound probe, if available.

\section{METHODS}

Begin by inserting the thermal imaging camera into the charging port of the smartphone. The vendorprovided thermography app is next selected. When the camera is turned on, a photo or video option may be chosen. With the latter positioned at a standard distance, about $70 \mathrm{~cm}$ from the flap itself ${ }^{[12]}$, images are observed and a thermogram taken as desired.

\section{RESULTS}

\section{Preoperative}

A thermal stress of the territory selected as the flap donor site is easily achieved by evaporation of an isopropyl alcohol spray accelerated with a portable fan [Figure 1]. A thermogram will confirm that this "cold challenge" is successful as darker colors on the color palette will be seen, implying lower skin temperatures [Figure 2]. During rewarming, "hot spots" appear that can be marked with a pen positioned as part of the thermal image [Figure 3]. These sites so rapidly delineated can then be further evaluated with the ubiquitous audible Doppler or color Duplex ultrasound to confirm the suspected presence of a perforator. A free flap can then be designed in the usual fashion as desired about those identified perforators.

\section{Intraoperative}

After the obligatory exploratory incision, if multiple possible perforator choices are found to exist, each in turn can be clamped temporarily with a microvascular clamp [Figure 4], and flap perfusion from each perforator assessed by evaluating the resulting thermogram [Figure 5]. If inadequate, perhaps more 


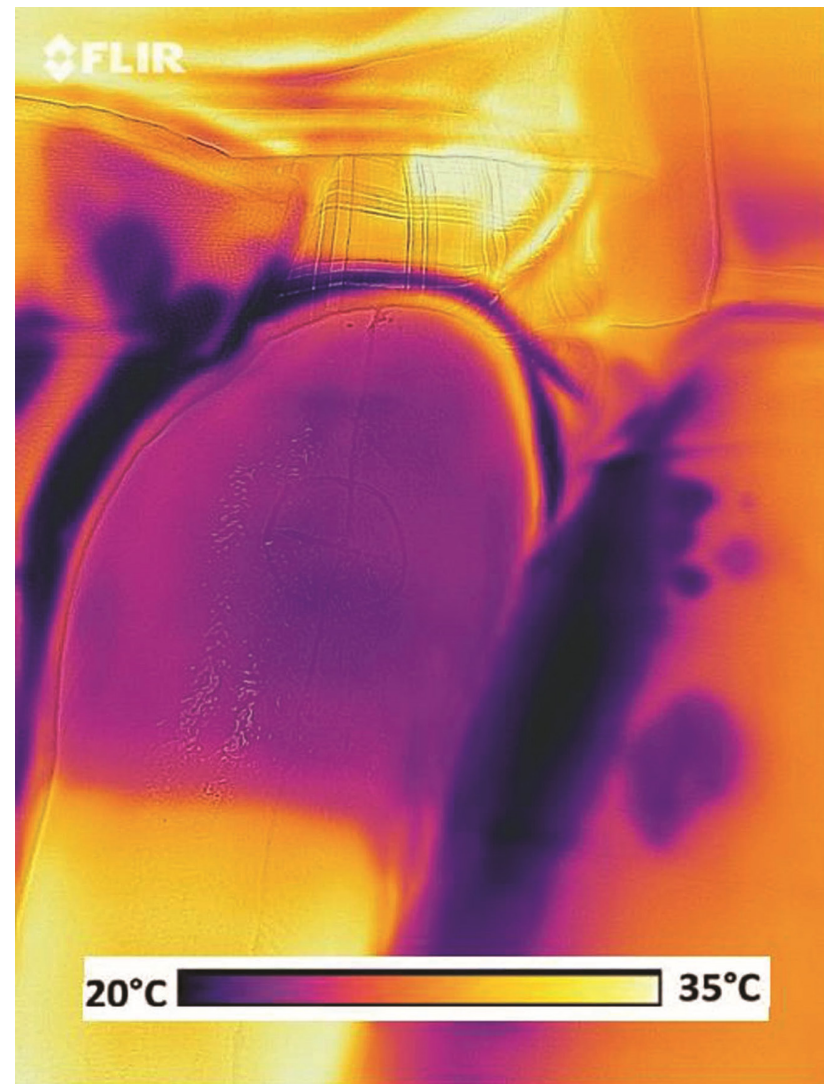

Figure 2. Preoperative: photograph of left thigh thermal image as seen after "cold challenge". Darker colors correspond to colder temperatures as seen on color bar below

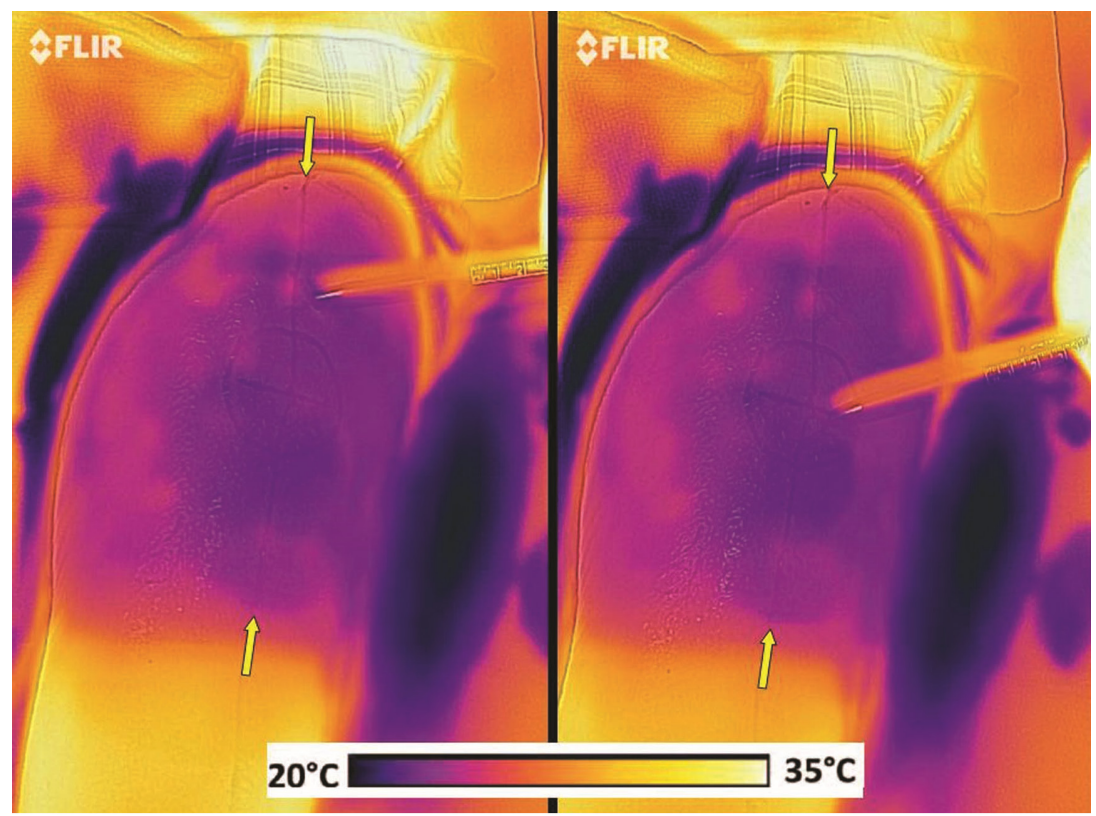

Figure 3. Preoperative: with rewarming, the brightest anterolateral thigh region "hot spot" denoted by marking pin held by assistant is observed proximal to a circle faintly seen drawn about midpoint of line (endpoints marked by yellow arrows) from anterosuperior iliac spine to superior lateral border of patella (left); and second "hot spot" in similar fashion seen more distal at center of that circle (right) 


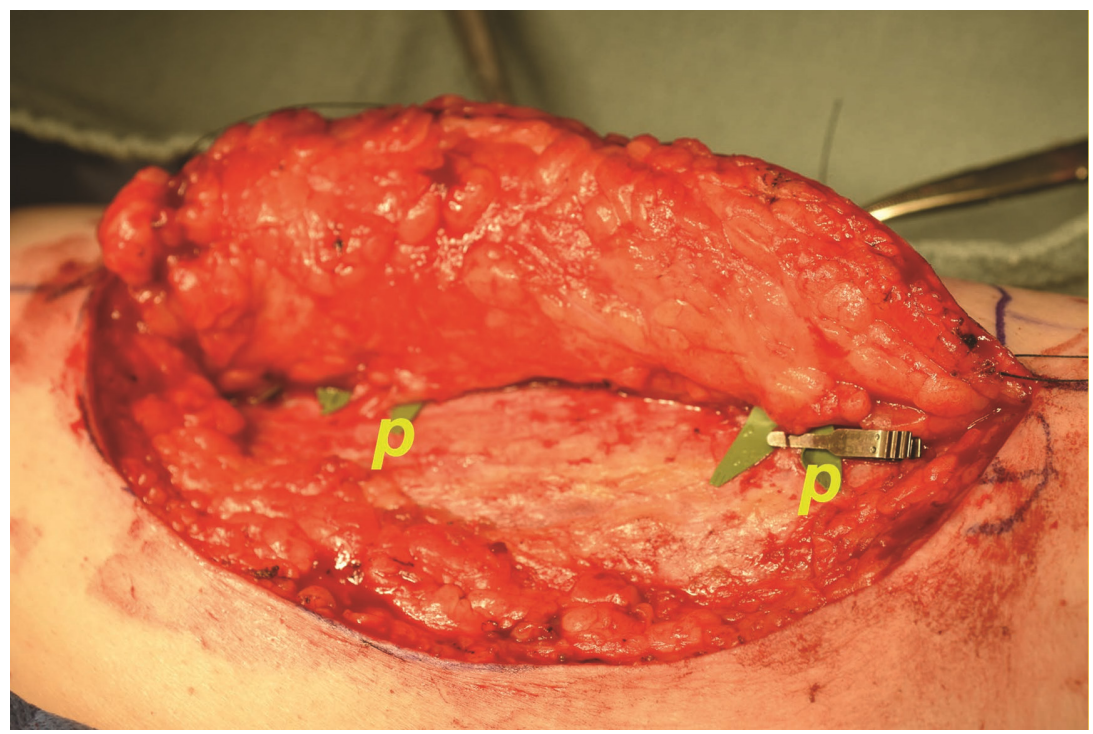

Figure 4. Intraoperative: exploratory incision revealed a perforator ( $p$ ) exactly at the two sites marked on the thermogram in Figure 3 (proximal thigh at left)

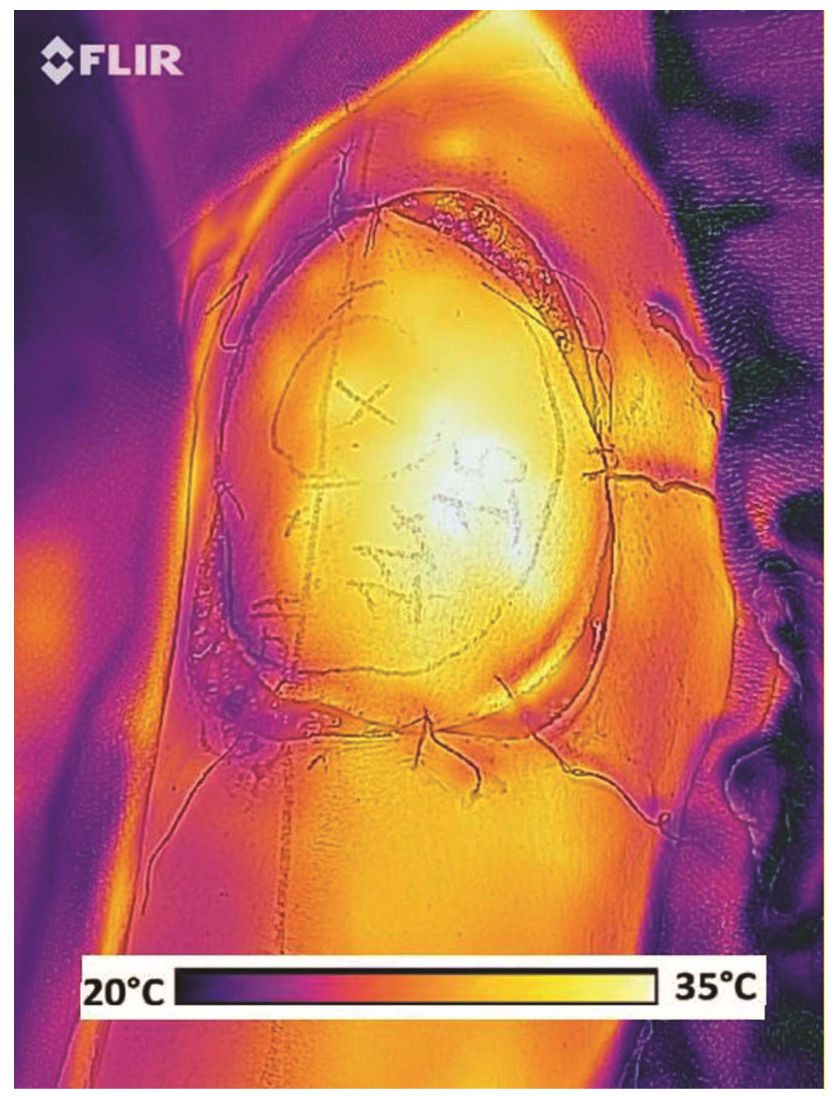

Figure 5. Intraoperative: thermogram with distal perforator clamped as in Figure 4 demonstrated virtually total anterolateral thigh flap perfusion via the proximal perforator alone, thus the second perforator could be discarded

than one perforator will need to be retained. Certainly, if the source pedicle of the flap itself is clamped, although the flap subjectively may appear well perfused, the corresponding thermogram will appropriately appear cool as expected [Figure 6]. Upon completion of the microanastomoses with flap revascularization, the flap should not only have a good appearance, but a correspondingly bright thermogram [Figure 7]. 


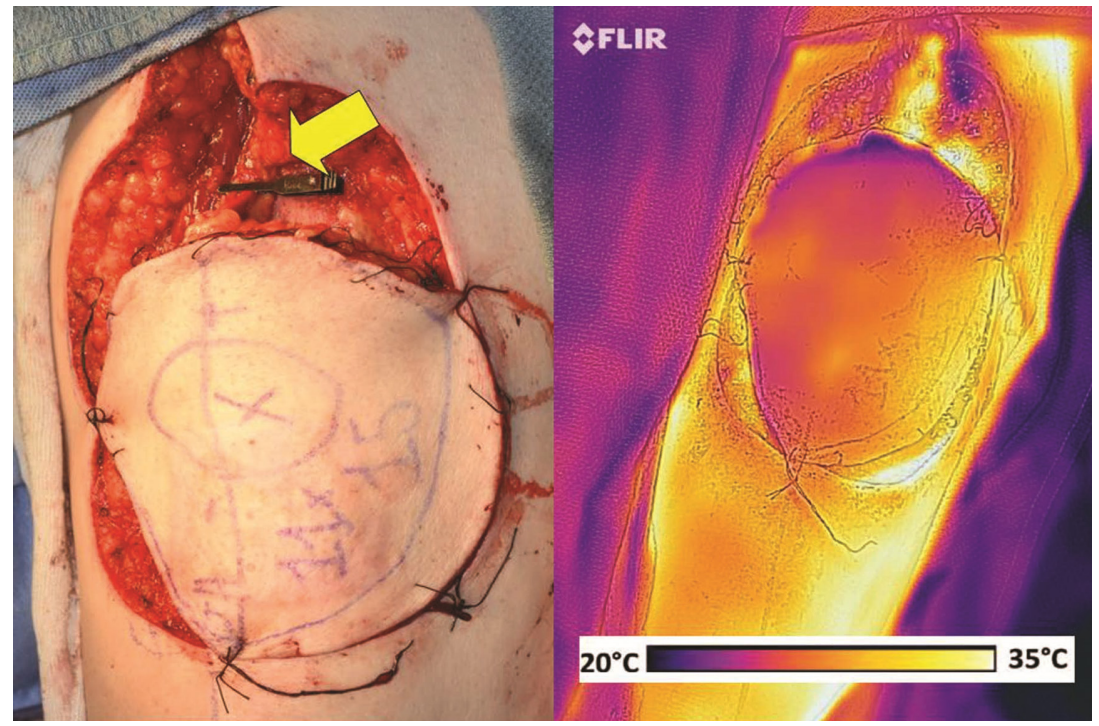

Figure 6. Intraoperative: subjectively, note "normal" appearance of anterolateral thigh flap, yet descending branch of lateral circumflex femoral source pedicle has been clamped (arrow) (left), but, as would be anticipated, the thermogram contradicts the observer's assessment as the entire flap is cool, since indeed there was no perfusion and so no radiative heat loss (right)

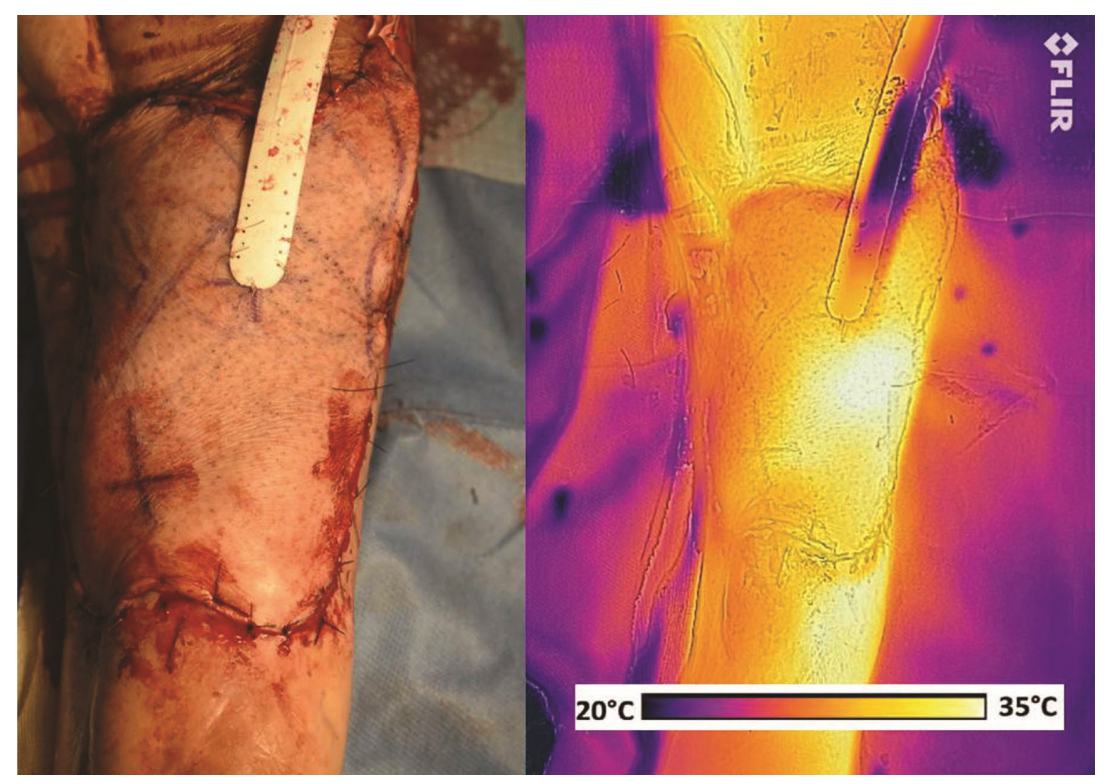

Figure 7. Intraoperative: anterolateral thigh flap inset on left lower leg (left), with thermogram confirming that revascularization was without compromise (right)

\section{Postoperative monitoring}

Routine monitoring protocols should always be followed. Maintenance of bright colors implying a warm flap as seen by the thermogram will confirm adequate perfusion and be consistent with satisfactory flow across the microanastomosis [Figure 8]. This, of course, will persist if successful long term [Figure 9].

A baseline thermal image at the time of completion of the procedure should always be available for comparison later while monitoring a free flap [Figure 10]. A change in the thermogram if the observed color is darker implies diminished flow. Venous congestion, with persistent arterial inflow to some degree, will result in a diffusely homogeneous thermogram [Figure 11]. This will be in distinction to a normal 


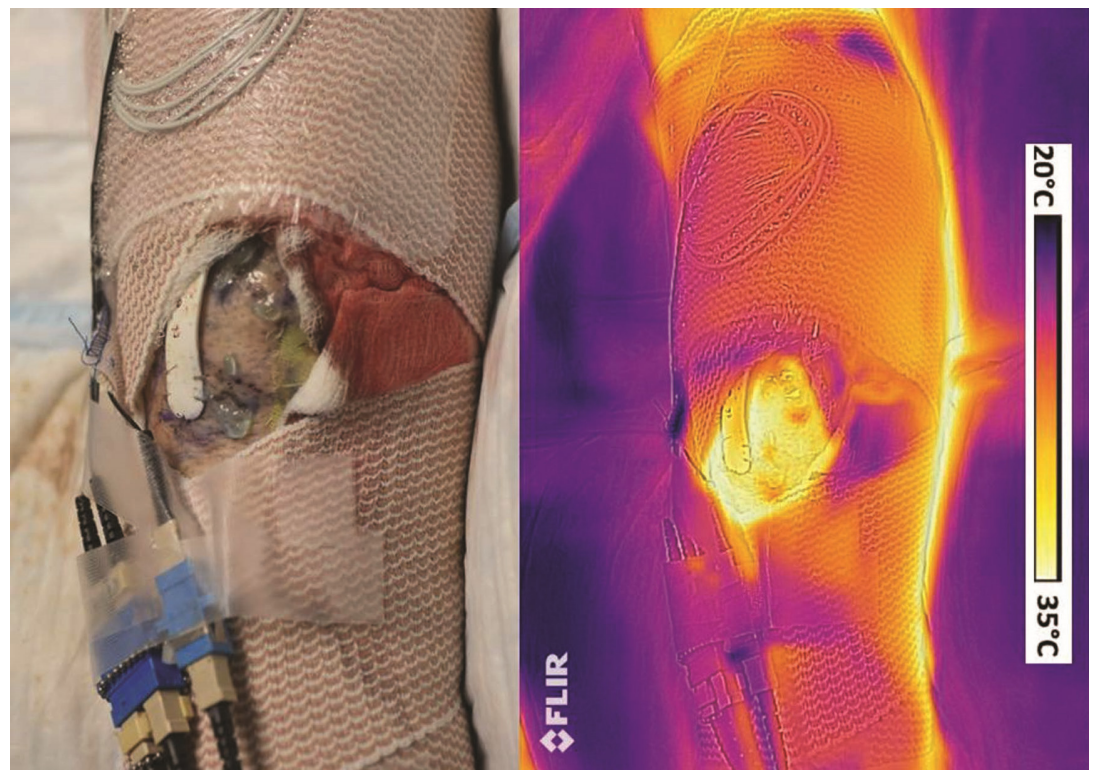

Figure 8. Postoperative monitoring: anterolateral thigh free flap appears satisfactory on inspection in dressing window on POD 2 (left). POD 2 thermogram shows variations of bright color pattern throughout flap, while dressings are dark, implying coolness, as they of course have no perfusion (right). POD: postoperative day

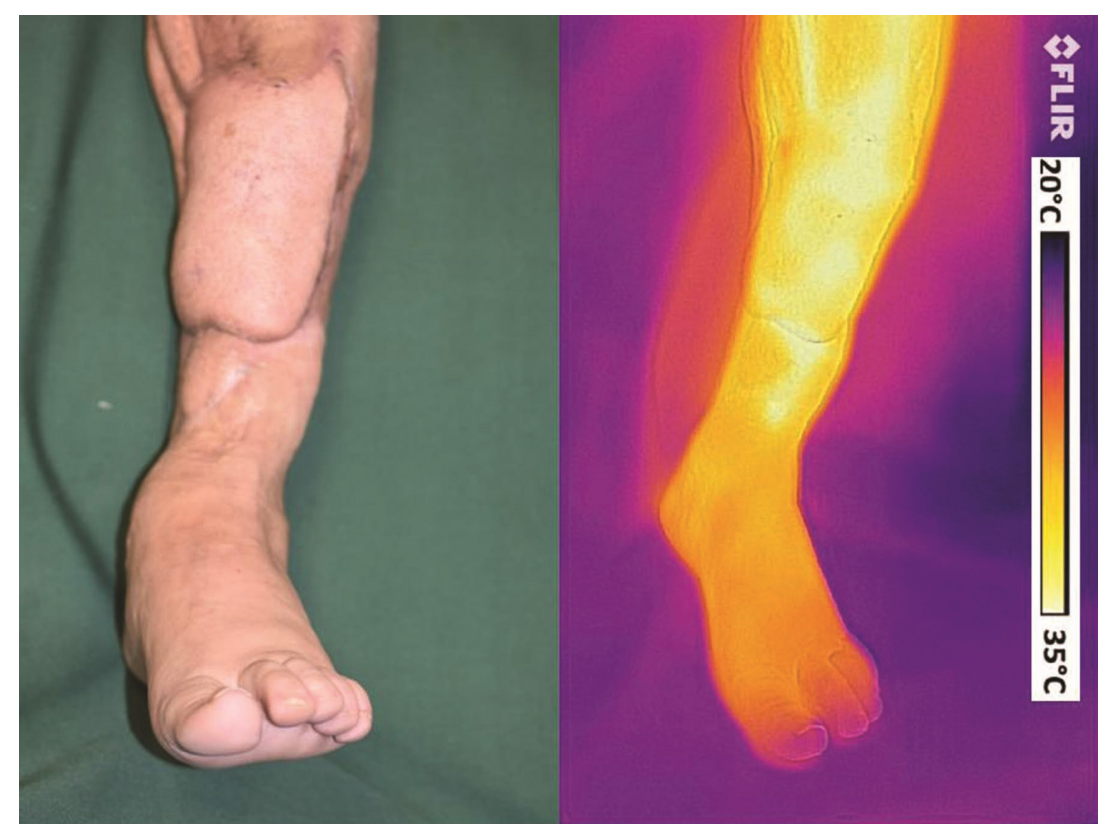

Figure 9. Postoperative monitoring: left leg anterolateral thigh free flap at POD 40 (left), and the POD 40 thermogram confirms flap adequately perfused and even warmer than toes (right). POD: postoperative day

perfusion pattern, as always present subtle differences in flow between encompassed vascular networks will be observed on the thermogram with some color variations throughout the flap [Figure 8]. Lack of inflow will result in a cold flap without any signs of perfusion [Figure 12].

\section{DISCUSSION}

Thermal imaging cameras have become incredibly inexpensive, thus, when attached to a smartphone, now anyone can assess free flap donor sites with virtually no learning curve ${ }^{[6,11]}$. Following the simple preceding 


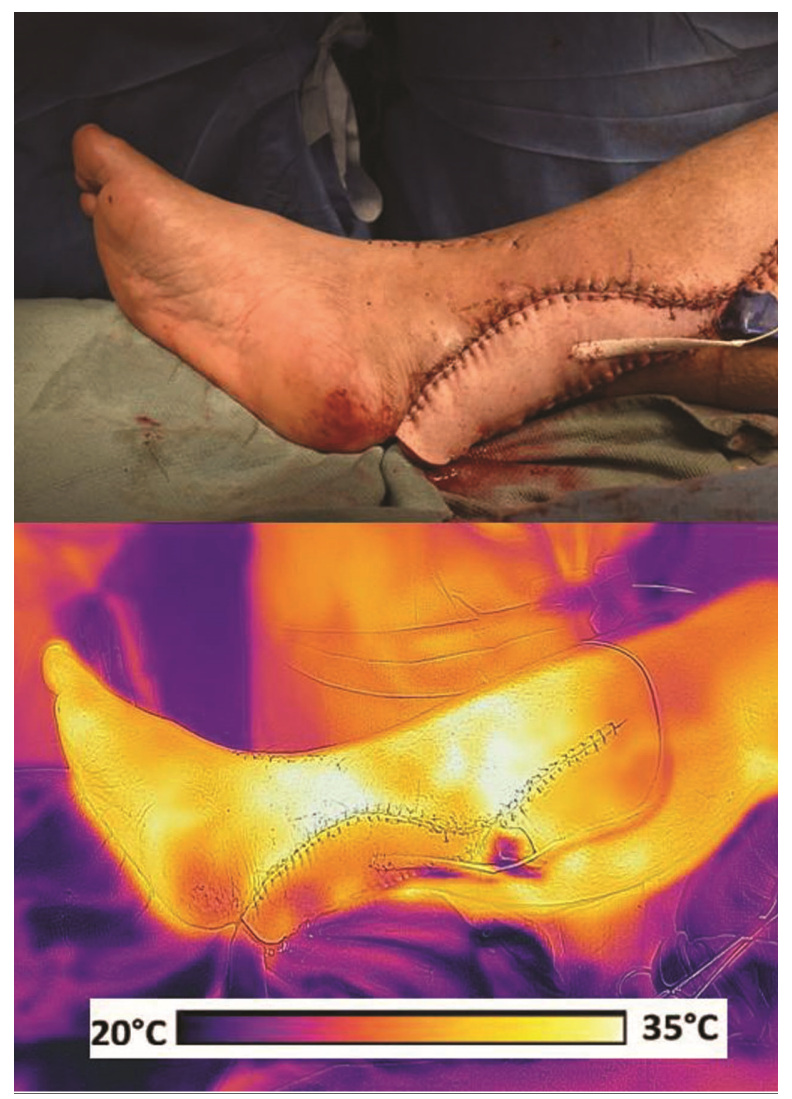

Figure 10. Postoperative catastrophe: achilles tendon rupture covered with anterolateral thigh free flap had good visual color as seen intraoperatively (top), and intraoperative thermogram confirmed a warm flap with good perfusion comparable to surrounding leg skin (bottom)

guidelines, thermography can assist in the identification of perforators to facilitate the preoperative design of a free perforator flap. A concordance study by Pereira et al. ${ }^{[6]}$ compared preoperative detection of perforators by smartphone thermography with CT angiography, and showed high accuracy with a sensitivity of $100 \%$ and specificity of $98 \%$. Recognized traditional imaging techniques for perforator identification in addition to CT angiography ${ }^{[15]}$ such as magnetic resonance angiography ${ }^{[16]}$, or color Duplex ultrasound ${ }^{[17]}$ remain reliable and sound alternatives, but may not be universally available. However, in contrast to thermography, all the aforementioned also may be expensive, perhaps require exposure to contrast media or ionizing radiation, and will be relatively time consuming ${ }^{[3,17,18]}$. Certainly, thermography as a complementary procedure, if for no other attribute, can be done quickly to allow more intense focus on "hot spots" for follow-up with the ubiquitous audible Doppler, or perhaps color Duplex ultrasound.

Thermography also offers many insights to provide effective intraoperative management, including what perforators may be satisfactory to retain or what portion of the flap will be expected to be viable. The adequacy of flap perfusion following revascularization or any compromise upon insetting can be determined without the expense or demand for indocyanine green angiography ${ }^{[19]}$. Finally, of course, the thermogram provides an additional means for postoperative monitoring. The same smartphone used to make the thermogram can be used to send these pictures wherever needed for corroboration. A thermogram is a near perfect monitor being simple to obtain, non-invasive, and accurate; however, it is not continuous and only semi-objective, as some interpretation of the color palette representing flap temperature is required. 


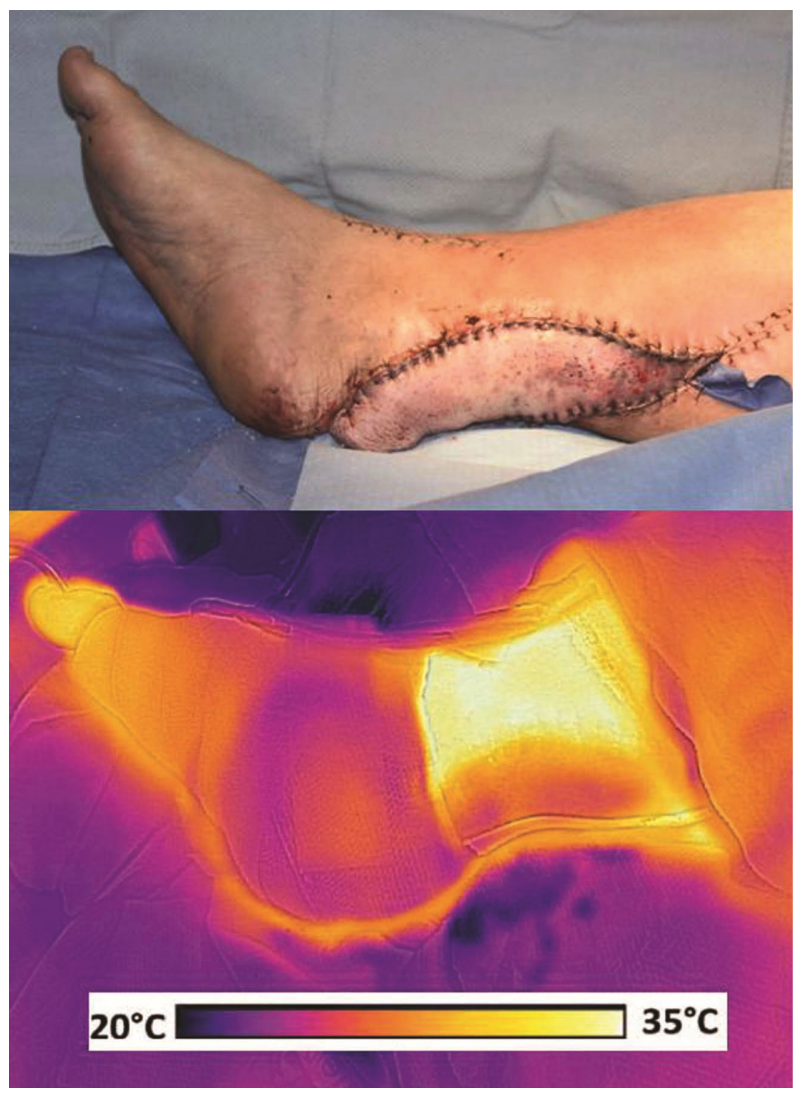

Figure 11. Postoperative catastrophe: violaceous hue of flap POD 1 (top). POD 1 thermogram of flap in dressing window had much darker color diffusely throughout than seen intraoperatively consistent with venous congestion (bottom). POD: postoperative day

Most studies to date using the principles of thermography have centered on detection of perforators of free flap donor sites or monitoring of microvascular tissue transfers ${ }^{[4,8,13,14,18]}$. Only two previous reports have used a smartphone for thermography ${ }^{[6,1]}$, and both for the same purpose as here reviewed in greater detail. There is no reason that the same advantages of thermography cannot also be applied to local perforator flaps as well ${ }^{[20,21]}$. Remember Georgescu et al. . $^{[22]}$ 's admonition that even local perforator flaps are microsurgical non-microvascular tissue transfers, and should be approached in a similar fashion as are free flaps using whatever resources are available.

An awareness of the limitations of thermal imaging cameras is also important. These can detect only the physiology due to alterations in surface body temperature, which is directly correlated to perforators; however, they cannot distinguish their morphology, thus there will be no recognition of the caliber, origin, or path of that perforator, which, after penetrating the deep fascia, could have an oblique course or diverge into multiple branches to result in multiple "hot spots" from a single perforator before reaching the $\operatorname{skin}^{[5,8]}$. Professional thermal cameras, being more sensitive than smartphones, are less likely to be misled by any background thermal interference or artifacts such as the presence of cutaneous veins or heat hollows ${ }^{[8]}$. In our experience, unlike with the professional thermal cameras, use of a smartphone has required a "cold challenge" to allow a thermal recovery to best determine the significance of "hot spots" in the preoperative detection of donor site perforators ${ }^{[3,5]}$. Note also that the smartphone visible light photograph will always be offset slightly from the digital thermogram [Figure 13 $]^{[7]}$. This must always be accounted for, especially if the exact location of perforators is essential. 


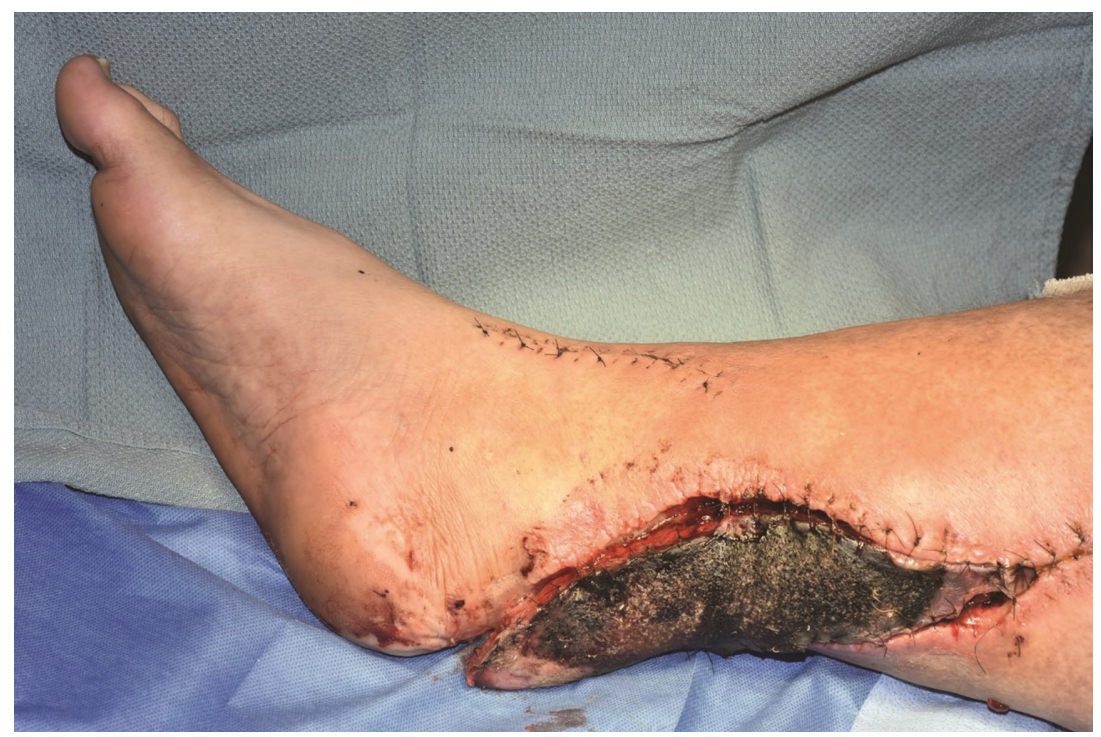

Figure 12. Postoperative catastrophe: on re-exploration, venous congestion due to a venous thrombosis could not be reversed, and leech therapy was unsuccessful for flap salvage

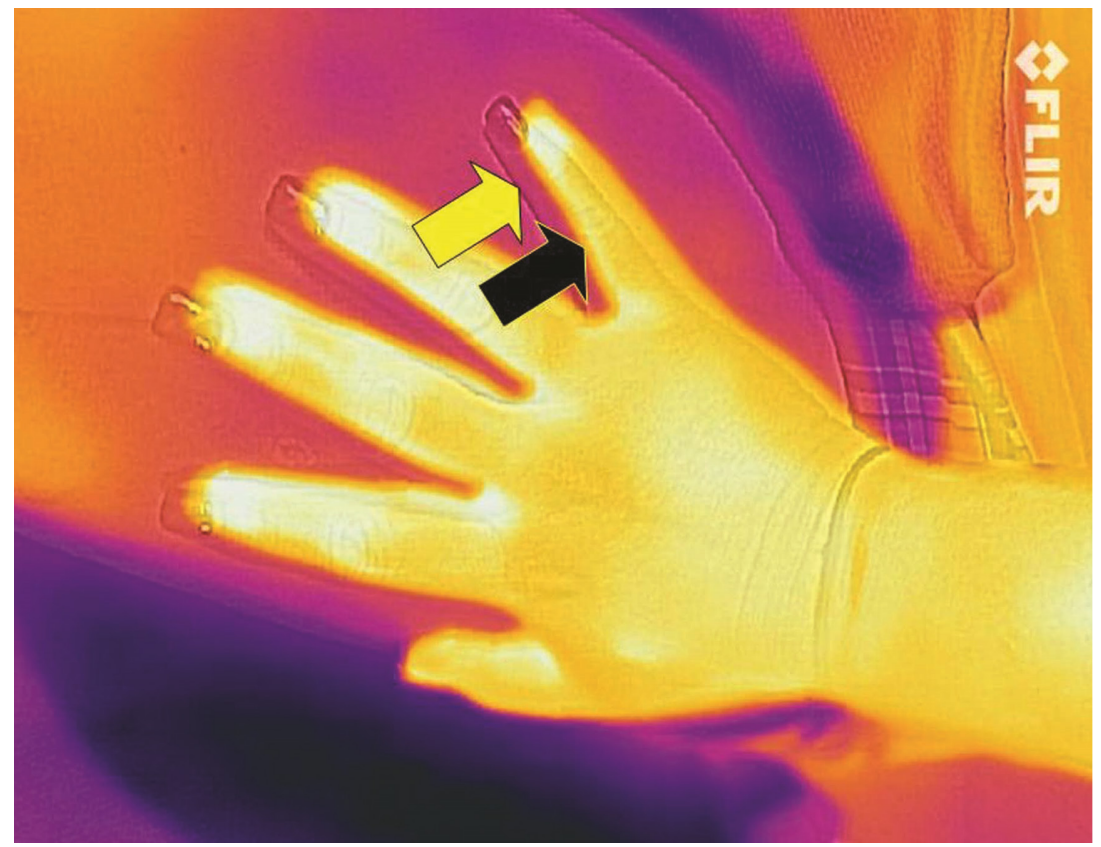

Figure 13. The observed thermal image (black arrow) can be offset from the visible camera image (yellow arrow) as seen here by almost $1 \mathrm{~cm}$

In conclusion, despite the many attributes and plausible detriments enumerated above, the value and the ultimate role of using a smartphone and an inexpensive commercial thermal imaging camera for thermography has yet to be fully determined. Applications will surely not only be for free perforator flaps, but also local perforator flaps, and maybe someday muscle flaps as well. The learning curve is short, thus acquisition of a smartphone and a thermal imaging camera should universally better permit safer free flap designs, provide additional intraoperative management insight, and even be another means for postoperative free flap monitoring. Perhaps with more experience, someday thermography will be more than just a complementary adjunct in the use of perforator flaps in general. 


\section{DECLARATIONS}

\section{Acknowledgments}

David C. Rice, B.S., P.E., St. Luke’s Hospital, Sacred Heart Division, Allentown, Pennsylvania, assisted with thermograms and flap harvest.

\section{Authors' contributions}

The author contributed solely to the article.

\section{Availability of data and materials}

Not applicable.

\section{Financial support and sponsorship}

None.

\section{Conflicts of interest}

The author declared that there are no conflicts of interest.

\section{Ethical approval and consent to participate}

Not applicable.

\section{Consent for publication}

Not applicable.

\section{Copyright}

(c) The Author(s) 2019.

\section{REFERENCES}

1. Koerner S, Adams D, Harper SL, Black JM, Langemo DK. Use of thermal imaging to identify deep-tissue pressure inury on admission reduces clinical and fianancial burdens of hospital-acquired pressure injuries. Adv Skin Wound Care 2019;32:312-20.

2. Theuvenet WJ, Koeyers GF, Borghouts MH. Thermographic assessment of perforating arteries: a preoperative screening method for fasciocutaneous and musculocutaneous flaps. Scand J Plast Reconstr Surg 1986;20:25-9.

3. de Weerd L, Mercer JB, Weum S. Dynamic infrared thermography. Clin Plast Surg 2011;38:277-92.

4. de Weerd L, Weum S, Mercer JB. The value of dynamic infrared thermography (DIRT) in perforator selection and planning of free DIEP flaps. Ann Plast Surg 2009;63:274-9.

5. Muntean MV, Strilciuc S, Ardelean F, Georgescu AV. Dynamic infrared mapping of cutaneous perforators. J Xiangya Med 2018;3:16.

6. Pereira N, Valenzuela D, Mangelsdorff G, Kufeke M, Roa R. Detection of perforators for free flap planning using smartphone thermal imaging: a concordance study with computed tomographic angiography in 120 perforators. Plast Reconstr Surg 2018;141:787-92.

7. Muntean MV, Achimas-Cadariu PA. Detection of perforators for free flap planning using smartphone thermal imaging: a concordance study with computed tomographic angiography in 120 perforators. Plast Reconstr Surg 2018;142:604e.

8. Tenorio X, Mahajan AL, Elias B, van Riempst JS, Wettstein, et al. Locating perforator vessels by dynamic infrared imaging and flow doppler with no thermal cold challenge. Ann Plast Surg 2011;67:143-6.

9. Saint Cyr M, Wong C, Schaverien M, Mojallal A, Rohrich RJ. The perforasome theory: vascular anatomy and clinical applications. Plast Reconstr Surg 2009;124:1529-44.

10. Sheena Y, Jennison T, Hardwicke JT, Titley OG. Detection of perforators using thermal imaging. Plast Reconstr Surg 2013;132:1603-10.

11. Hardwicke JT, Osmani O, Skillman JM. Detection of perforators using smartphonethermal imaging. Plast Reconstr Surg 2016;137:39-41.

12. Pereira N. Reply: detection of perforators for free flap planning using smartphone thermal imaging: a concordance study with computed tomographic angiography in 120 perforators. Plast Reconstr Surg 2018;142:605e.

13. de Weerd L, Mercer JB, Setsá LB. Intraoperative dynamic infrared thermography and free-flap surgery. Ann Plast Surg 2006;57:279-84.

14. Itoh Y, Arai K. Use of recovery-enhanced thermography to localize cutaneous perforators. Ann Plast Surg 1995;34:507-11.

15. Masia J, Kosutic D, Clavero D, Larrañaga J, Vives L, et al. Preoperative computed tomographic angiogram for deep inferior epigastric artery perforator flap breast reconstruction. J Reconstr Microsurg 2010;26:21-8.

16. Masia J, Kosutic D, Cervelli D, Clavero JA, Monill JM, et al. In search of the ideal method in perforator mapping: noncontrast magnetic resonance imaging. J Reconstr Microsurg 2010;26:29-35.

17. Hallock GG. Doppler sonography and color duplex imaging for planning a perforator flap. Clin Plast Surg 2003;30:347-57. 
18. Chubb D, Rozen WM, Whitaker IS, Ashton MW. Digital thermographic photography ("thermal imaging") for preoperative perforator mapping. Ann Plast Surg 2011;66:324-5.

19. Holm C, Tegeler J, Mayr M, Becker A, Pfeiffer UJ, et al. Monitoring free flaps using laser-induced fluorescence of indocyanine green: a preliminary experience. Microsurgery 2002;22:278-87.

20. Muntean MV, Ardelean F, Strilciuc S, Pestean C, Georgescu AV, et al. Flap warming improves intraoperative indocyanine green angiography (ICGA) assessment of perfusion. an experimental study. J Plast Reconstr Aesthet Surg 2019;72:1150-6.

21. Pereira N, Hallock GG. SmartPhone thermagraphy for lower extremity local flap perforator mapping. J Reconstr Microsurg 2020;pub pend.

22. Georgescu AV, Matei I, Ardelean F, Capota I. Microsurgical non-microvascular flaps in forearm and hand reconstruction. Microsurgery 2007;27:384-94. 